\title{
NOVEL MULTI-TENSOR ESTIMATION FOR HIGH- RESOLUTION DIFFUSION TENSOR MAGNETIC RESONANCE IMAGING
}

\author{
Inas A. Yassine*, Abou-bakr M. Youssef, Yasser M. Kadah. \\ Systems and Biomedical Department, Faculty of engineering Cairo University.
}

\section{ABSTRACT}

\begin{abstract}
The estimation of diffusion tensors in diffusion tensor imaging (DTI) is based on the assumption that each voxel is homogeneous and can be represented by a single tensor. As a result, estimation errors arise particularly in voxels with partial voluming of white matter or gray matter with cerebrospinal fluid (CSF) and voxels where fibers cross. Several authors have explored the possibility of solving for multiple tensors. Several authors analyzed the problem from the point of view of the number of unknowns and concluded that the solution is rather difficult due to the large number of unknowns and the nonlinearity of the equations. However, their approach was only helpful in eliminating some of the sources of artifacts in the DTI data but offered only a qualitative description of the model components. We implemented three strategies gradient, Differentiation and exhaustive algorithms to solve and compare between their estimations at various conditions of signal to noise ratios (SNR).
\end{abstract}

\section{INTRODUCTION}

Diffusion tensor imaging (DTI) is a non-invasive method of characterizing tissue micro-structure. Diffusion imaging attempts to characterize the manner by which the water molecules within a particular location move within a given amount of time. Using a simple pulse gradient spin echo (PGSE) imaging sequence, it is possible to obtain a change of the MR signal that is related to the diffusivity of water in a certain direction ${ }^{1}$. The advantage of this modality lies in the fact that the changes in water diffusion, produced by alterations in brain biochemistry, can be observed on diffusion weighted (DW) images long before the effects of ischemic injury can be seen on conventional T1, or T2 weighted images ${ }^{2}$. Measurement of the diffusion tensor (D) within a voxel enables the mobility of water to be characterized along orthotropic axes, allows a macroscopic voxel-averaged description of fiber structure, orientation $^{3}$ and fully quantitative evaluation of the microstructural features of healthy and diseased tissue ${ }^{2}$. D is estimated using a set of diffusion-weighted images ${ }^{4}$. The basic techniques in diffusion tensor imaging attempt to characterize the 3-D diffusion phenomena in terms of a 3-D Gaussian probability distribution ${ }^{3}$. Therefore, such representation is sufficient in terms of a $3 \times 3$ symmetric tensor, or the so-called "cigar-shaped" diffusion tensor representation. This tensor is usually computed using a 3-D sampling of the b-space of measurement in the diffusion-weighted experiment ${ }^{5}$. While requiring only six equations to completely determine the diffusion tensor, more measurements are usually obtained and a least-squares solution is calculated for the tensor.

Recent studies revealed several deviations from this simplified scenario, because the tensor model is incapable, however, of resolving multiple fiber orientations within an individual voxel. This shortcoming of the tensor model stems from the fact that the tensor possesses only a single orientational maximum, i.e., the major eigenvalue of the diffusion tensor. At the millimeter-scale resolution typical of DTI, the volume of cerebral white matter containing such intravoxel orientational heterogeneity (IVOH) may be considerable given the widespread divergence and convergence of fascicles. The abundance of IVOH at the millimeter scale can be further appreciated by considering the ubiquity of oblate "pancake-shaped" diffusion tensors in DTI, a hypothesized indicator of IVOH. Given the obstacle that IVOH (particularly fiber crossing) poses to white matter tractography algorithms, we sought to determine whether high angular resolution, high $b$-value diffusion gradient sampling could resolve such intravoxel heterogeneity ${ }^{6}$.

* Inas_Yasine(@)k-space.org 
Several authors reported this non-mono-exponential behavior for the diffusion-induced attenuation in brain tissue in water and NAA signals. Their study was based on a 1-D diffusion experiment and the results were fitted to bi- or triexponential functions. They indicated that high $b$ values reveal more complex behavior and therefore their fit depended on the value of $b$ used in the measurement.

Basser et al $^{7}$ discussed the possibility of mixture modeling of diffusion. Even though they indicated that this would present a more complete representation of the process, they argued that there too many issues that need to be resolved before such modeling can be performed in practice. In particular, their hypothetical discussion indicated that such modeling would require a large amount of data to enable the estimation of model parameters and involve the computation of too many parameters. They suggested also that several problems had to be addressed in such experiments that included optimization of diffusion gradient directions and model order selection. They concluded that this area had many aspects that were yet to be investigated. Hsu et al. ${ }^{8}$ proposed a two-compartment model for the diffusion in fibers of the myocardium. They reported two fast and slow components in their study while assuming a slow-exchange process between the two. Inglis et al. ${ }^{9}$ reported biexponential diffusion tensor measurements. They hypothesized that these components may represent the intra- and extra-cellular components in tissues. Clark et al. ${ }^{10}$ reported variations of the apparent diffusion coefficient with the value of diffusion time. Their hypothesis was that such variations are imporiant indicators of restricted flow, which present a potentially large diagnostic value. In a later study by the same group, Clark et al. ${ }^{11}$ reported results of a two-tensor model for diffusion in the human brain. They measured the parameters of a mixture model composed of two weighted tensors. Their results indicated the presence of fast and slow diffusion components, and that each can be modeled by a unique tensor. They indicated that the use of high $b$ value was essential to reveal the slow component of diffusion.

Frank ${ }^{12}$ reported a method for identifying the anisotropy in high angular resolution diffusion-weighted (HARD) imaging data without computing the actual tensor. Another study by the same author ${ }^{13}$ developed a methodology for characterizing HARD data by decomposition into spherical harmonics. This approach allowed several modes of diffusion to be decomposed into separate channels that are different from those for eddy current artifacts. He studied the case of two fibers under different conditions. The proposed an extension of his method to characterize multiple fiber scenarios. Given the complexity of such situations and the limitations on the spherical harmonics definitions in terms of rotations only, this might not be practical in many cases. Wang Zhan et al. ${ }^{14}$ introduce a study to develop an alternative based on getting a circular spectral decomposition technique. The basic premise of this method is to determine the $\mathrm{ADC}$ values on the unit circle spanned by the major and median eigenvectors of the diffusion tensor, and then apply a ID Fourier-transform onto this circle They assumed that the effective diffusion anisotropy of a single fiber typically exhibits a cylindrical symmetry about its longitudinal axis, and therefore the scalar ADC distribution of a single-fiber system The plane embedding this sampling circle should include the fiber's longitudinal axis that corresponds to the major eigenvector. The circular ADC distribution of a single fiber is comprised of a constant offset equal to the average of the major and median eigenvalues, plus a sinusoid function with an angular frequency of 2 . The partial volume effects were been taken into account if two fibers occupy the same voxel. Under the assumption that there is no exchange between the two diffusion compartments and there is equal signal contribution from the two fibers. Tuch et $a l^{6}$ noted that the DTI measurements could only resolve the imaging situations where the white matter fibers are strongly aligned. They presented evidence from high angular resolution diffusion measurements to show that the diffusion process can be modeled as independent mixture of ideal diffusion processes. They presented results for the case of a mixture of two diffusion tensors. The methodology used to obtain the mixture parameters was based on minimizing an error function using gradient descent technique. Kadah et al. ${ }^{15}$ utilize a multi-compartmental model to represent the physical make-up of imaging pixels. Based on an analytical expression of apparent diffusion tensor, the mixture model parameters are calculated using global nonlinear least squares methods. Given the characteristics of axonal membranes, the diffusion of water inside each axon is preferred along the direction of the axon than across it.

Tournier et al. ${ }^{16}$ have proposed a technique that is able to estimate the distribution of fiber orientations directly from the diffusion-weighted data, using the concept of spherical deconvolution. This technique requires a resporlse function and associated low-pass filter (to mitigate the effects of noise). The optimal shape for these functions will in general depend on factors such as the SNR, the number of diffusion-encoding directions used, the $b$-value, and physiological factors such as the level of maturation of the brain. Their objective was estimating and optimizing 
these functions, using a minimum entropy concept, to ensure that the results provided by the technique are robust and reproducible .In addition, they proposed a normalized version of the entropy as a natural alternative measure of anisotropy for non-tensor derived fiber orientation distributions. Another method was proposed by the same group ${ }^{17}$ to estimate the fiber orientation distribution (FOD) directly from high angular resolution diffusion-weighted data, using the concept of spherical deconvolution. This technique was used to estimate the FOD adequately even in regions containing multiple fiber populations, where diffusion tensor based techniques are known to be deficient. They apply the bootstrap concept to the spherical deconvolution method to assess the precision of the various estimated fiber orientations. The spherical deconvolution technique estimates the FOD by assuming a response function (the diffusion profile for a typical fiber bundle) and deconvolving it from the diffusion-weighted signal profile over spherical coordinates. The response function and filter parameters used for the spherical deconvolution were estimated from the data themselves using a minimum entropy principle.

Observing that the diffusion along nerve fibers tend to be significantly larger that that in other directions (Basser et $a .^{18}$ ), track fiber directions were computed from diffusion tensor data. The basic idea is to eigen-decompose the diffusion tensor and use the eigenvector corresponding to the largest eigenvalue as the most likely fiber direction in a given pixel. This simplistic representation of the problem is often challenged by real data where fiber direction heterogeneity is common. As a result, several studies indicated practical problems with this approach (cf. Mori and van $\mathrm{Zij}^{19}$ ). In general, the heterogeneity results in situations where the direction of the fiber cannot be determined. For example, when the diffusion ellipsoid takes the form of a pancake shape rather than a cigar shape. In such cases, the tracking algorithms terminate at these points resulting in undesired disconnections in the resulting fiber tracks. Poupon $e t$ al. ${ }^{20}$ reported problems with the tracking results when crossing fibers are encountered. They proposed a methodology whereby some a priori knowledge about the fiber tracks are embedded in the fiber tracking procedure to regularize the calculated fiber directions. Similar regularization was proposed by Bammer $e t$ al $^{21}$ where they used a continuous representation based on B-spline modeling.

After the diffusion tensor principal directions are determined, the problem of finding the fiber paths from the diffusion vector field (or the so called tractography problem) is a challenging one. Several techniques were proposed to do that based on line propagation or energy minimization implemented in different manners to try to overcome the limitations posed by noise, limited resolution and direction heterogeneity of fibers within the resolution cell (cf. Mori and van $\mathrm{Zijl}^{19}$, Lori $e t$ al. $^{22}$ ). The general limitations of the available techniques are the speed of axonal reconstruction, robustness in the presence of anisotropy in the diffusion tensor (e.g., the pancake shaped tensor case), as well as the difficulty of validating the outcome and the different constraints used in this processing. None of the available tracking methods provided a vision for how tracking should be done in case axonal intersections are resolved within resolution cells. By comparison to the conventional situations, this problem seems far more challenging since it involves adding another dimension to the original formulation representing axonal anisotropy.

The goal of this work is to simulate the three methodologies Tuch et al. ${ }^{6}$, Differentiation and Kadah et al. ${ }^{15}$ to solve multi-compartmental model to represent the physical make-up of imaging pixels. Based on an analytical expression of apparent diffusion tensor, the mixture model parameters are calculated at different Signal to noise Ratios and compared with each other.

\section{METHODS}

\subsection{Problem formulation}

Assuming Gaussian diffusion, the diffusion signal from a single diffusion compartment is given by:

$$
E\left(q_{k}\right)=\exp \left(-q_{k}^{T} D q_{k} \tau\right)
$$

where $E\left(\mathbf{q}_{k}\right)$ is the normalized diffusion signal magnitude for the diffusion gradient wave-vector $\mathbf{q}_{k}=\gamma \delta \boldsymbol{g}_{k} \gamma$ is the gyromagnetic ratio, $\delta$ is the diffusion gradient duration. $\mathbf{g}_{\mathrm{k}}$ is the $k$ th diffusion gradient, $\tau$ is the effective diffusion time, and $\mathbf{D}$ is the apparent diffusion tensor . To model multiple compartments, if we assume that: the inhomogeneity consists of a discrete number of homogeneous region;; the regions are in slow exchange, i.e., separated by a 


\section{The $23^{\text {rd }}$ National Radio Science Conference (NRSC 2006) \\ March 14-16, 2006 \\ Faculty of Electronic Engineering, Menoufiya University, Egypt.}

distance much greater than the diffusion mixing length; and the diffusion within each region is Gaussian, i.e., fully described by a tensor, then we can express the diffusion function as a finite mixture of Gaussian

$$
E\left(q_{k}\right)=\sum_{i} f_{i} \exp \left(-q_{k}^{T} D q_{k} \tau\right)
$$

Consider the problem of estimating the composition of a voxel with two distinct components (without loss of generality). In this case, the number of unknowns to fully describe the model is 13 (2 symmetric tensors and their partial volume ratios that add to one).

$$
E\left(q_{k}\right)=f_{1} \exp \left(-q_{k}{ }^{T} D_{1} q_{k} \tau\right)+\left(1-f_{1}\right) \exp \left(-q_{k}{ }^{T} D_{2} q_{k} \tau\right)
$$

Unlike the problem of estimating a single tensor, the equations here are nonlinear. The attenuation equation for each tensor resembles a sample of a 3D Gaussian function with a covariance matrix equal to the diffusion tensor evaluated at a point determined by the diffusion gradient direction at a radius equal to the square root of the b-value. Hence, the problem of estimating multiple tensors becomes one of 3D Gaussian mixture modeling from samples determined by the diffusion gradient vector sampling. This estimation problem is nonlinear and therefore only iterative estimation methods have been proposed in the literature. Given the convergence issues associated with such methods and their generally high computational burden, another more stable strategy is needed to solve this problem in practice. Note that for any given parameter estimation accuracy, there exists a finite number of possible solution that are determined by the a priori information about parameter ranges and the desired accuracy. Hence, the problem of finding the solution to this problem amounts to a combinatorial optimization problem. This means that a globally optimal solution can be found by exhaustive search or one of the more efficient random search strategies such as simulated annealing or genetic algorithms. Nevertheless, the computational effort involved in such techniques is prohibitive.

We tried to estimate a model of two tensors using the three introduced methods. The first was presented by Tuch $e t$ $a{ }^{6}{ }^{6}$ who were able to detect diffusion signals with multiple discrete maxima/ minima as a function of gradient orientation, indicating the presence of multiple underlying fiber populations. The second is based on the differentiation of the signal with respect to the $b$ values. And the third was presented by Kadah et al. ${ }^{15}$ who project the problem into a number of $1 D$ problems and then synthesizes the solution to the original problem from the $1 D$ solutions, their problem can be simplified even further by utilizing a sampling strategy in such a way to convert the problem into the sum of two exponentials.

\subsection{The gradient algorithm ${ }^{6}$}

They use the traditional method for solving Gaussian mixture problems of this type is the expectation maximization (EM) algorithm. However, they needed to solve the mixture problem with physiological constraints on the eigenvalues. Given the inability of the EM algorithm to handle such hard constraints, a gradient descent scheme was employed with multiple restarts (six restarts maximum) to solve the mixture model by solving the eigenvectors and volume fractions that give the lowest error between the predicted and observed diffusion. The eigenvalues of the individual tensors can be specified a priori or restricted to a particular range in order to prevent the algorithm from over fitting with unphysiological eigenvalues. Multiple restarts were employed to prevent the algorithm from settling on local minima. Approximately half of the iterations found the global minimum. The eigenvalues were specified at $\left(\lambda_{1}, \lambda_{2}, \lambda_{3}\right)=(1.5,0.4,0.4) \mu \mathrm{m}^{2} / \mathrm{ms}$ based on reported normal values. The eigenvalues were preset in order to prevent the individual tensor fits from assuming oblate forms.

The error function to be minimized is

$$
x=\sum_{k}\left(\hat{E}\left(q_{k}\right)-E\left(q_{k}\right)\right)^{2}=\sum_{k}\left(\sum_{j} f_{i} \hat{E}\left(q_{k}\right)-E\left(q_{k}\right)\right)^{2},
$$

where $\hat{E}$ is the predicted diffusion signal based on the multi tensor model, $\hat{E}_{j}\left(q_{k}\right)$ is the predicted diffusion signal from compartment $j$ (Eq. [1]), and $E$ is the observed diffusion signal. To ensure that the rolume fractions are 
properly bounded $(f i \in[0,1])$ and normalized $\left(\sum_{i} f_{i}=1\right)$ the volume fractions are calculated through the soft-max transform.

$$
f_{i}=\frac{\exp \eta_{i}}{\sum_{i} \exp \eta_{i}}
$$

The tensors $\mathrm{D}_{j}$ are parameterized in terms of the Euler angles $\alpha_{j}^{i}$

The gradient with respect to the Euler angles is

$$
\frac{\partial x}{\partial \alpha_{j}^{i}}=-\sum_{k}\left(\hat{E}\left(q_{k}\right)-E\left(q_{k}\right)\right) f_{i} \hat{E}_{j}\left(q_{k}\right) q_{k}{ }^{T}\left(\frac{\partial R_{j}}{\partial \alpha_{j}^{i}} \Lambda_{j} R_{j}^{T}+R_{j} \Lambda_{j} \frac{\partial R_{j}^{T}}{\partial \alpha_{j}^{i}}\right) q_{k}{ }^{T},
$$

where $R_{j}$ is the column matrix of eigenvectors and $\Lambda$, is the diagonal matrix of eigenvalues for tensor $\mathbf{D} j$.

The gradient with respect to the volume fraction parameters is

$$
\frac{\partial x}{\partial \eta_{i}}=\frac{\exp \eta_{i}}{\left(\sum_{i} \exp \eta_{i}\right)^{2}} \sum_{k}\left[\left(\hat{E}\left(q_{k}\right)-E\left(q_{k}\right)\right) \sum_{i}\left(1-\delta_{i j}\right)\left(\hat{E}\left(q_{k}\right)-E\left(q_{k}\right)\right) \exp \eta_{i}\right],
$$

where $\delta^{\mathrm{ij}}=1$ if $i=j$, and 0 if $i \neq j$.

\subsection{The Differentiation algorithm}

We developed this method after the observing that the measurements in diffusion tensor imaging are usually obtained for uniformly distributed values of $b$. Recalling that the Attenuation values are direct functions of the square root of $b$, the above formula can be simplified for this case such that,

Where $\tau_{i}=1 /\left(q_{k}{ }^{T} D_{i} q_{k}\right)$

$$
E(b)=f_{1} \exp \left(-b / \tau_{1}\right)+\left(1-f_{1}\right) \exp \left(-b / \tau_{2}\right),
$$

Hence, our problem is now to estimate exponentially decaying components, which is a rather common problem in many fields. In particular, we can describe the system described by $E(b)$ using a homogeneous second-order differential equation in the form,

Where

$$
E^{\prime \prime}(b)+a_{1} E^{\prime}(b)+E(b)=0,
$$

$$
E^{\prime}(b)=-\frac{f_{1}}{\tau_{1}} \exp \left(-b / \tau_{1}\right)-\frac{\left(1-f_{1}\right)}{\tau_{2}} \exp \left(-b / \tau_{2}\right), E^{\prime \prime}(b)=\frac{f_{1}}{\tau_{1}^{2}} \exp \left(-b / \tau_{1}\right)+\frac{\left(1-f_{1}\right)}{\tau_{2}{ }^{2}} \exp \left(-b / \tau_{2}\right),[10]
$$

Since the values of $E(b)$ are available for several values of $b$ and that its first and second order derivative can be obtained numerically from these values using the forward or central numerical differentiation formulas or using the frequency domain method using the differentiation property of the Fourier transform. In our simulations, we used the central numerical differentiation we can formulate a linear system to estimate the coefficients of the differential equation as,

$$
\left[\begin{array}{cc}
E^{\prime}\left(b_{1}\right) & E\left(b_{1}\right) \\
E^{\prime}\left(b_{2}\right) & E\left(b_{2}\right) \\
\vdots & \vdots \\
E^{\prime}\left(b_{n}\right) & E\left(b_{n}\right)
\end{array}\right]\left[\begin{array}{l}
a_{1} \\
a_{2}
\end{array}\right]=-\left[\begin{array}{c}
E^{\prime \prime}\left(b_{1}\right) \\
E^{\prime \prime}\left(b_{2}\right) \\
\vdots \\
E^{\prime \prime}\left(b_{n}\right)
\end{array}\right],
$$

Once the coefficients of the equations are computed, the second-degree polynomial characteristic equation is solved to obtain the roots corresponding to the exponential factors. Then, it is straight forward to compute the magnitudes from solving the linear equations obtained by substituting the estimated variances. Besides the important simplification in separating between the problems of estimating the exponential factors and the magnitudes. 


\section{The $23^{\text {rd }}$ National Radio Science Conference (NRSC 2006) \\ March 14-16, 2006 \\ Faculty of Electronic Engineering, Menoufiya University, Egypt.}

\subsection{The Exhaustive algorithm ${ }^{15}$}

Instead of attempting the solution in the $14 \mathrm{D}$ space of this problem, this method projects the problem into a number of $1 \mathrm{D}$ problems and then synthesizes the solution to the original problem from the $1 \mathrm{D}$ solutions. for a projection direction given by angles $\theta$ and $\phi$ with the $x$ and $y$ axes respectively and where $D_{i}$ is the diffusion tensor for one of the components. Moreover, the problem can be simplified even further by utilizing a sampling strategy in such a way to convert the problem into the sum of two exponentials. This problem is solved using a robust strategy in which the exponential decay constants are estimated using exhaustive search and the magnitude functions are estimated using linear system solution based on the choice of the decay constants. Given that the range of decay constants for human applications is rather limited, this strategy has superior speed to other strategies based on nonlinear least squares methods while offering the global solution to the problem. Once the 1D model is estimated, it can be used to provide an equation for eack diffusion tensor separately as identified by its partial volume ratio. After sufficient equations are collected, the equations for each tensor are solved independently to obtain the tensor. In case of a 3-tensor model, the same procedure is followed at an additional computational cost that varies linearly with the number of tensors. The computed tensors are used to compute a new estimate of the component partial volume ratios based on the whole data set rather than each projection separately. Given that these ratios are the most affected by noise, the estimation process is started again with this new estimate plugged in for all projections and a new solution is estimated. This process is repeated until the partial volume ratio stabilizes.

\subsection{Parameters Estimations}

The influence of noise on the estimates of the two tensor model was calculated using the three precedent defined algorithms was studied by Monte Carlo computer simulation, written using Matlab. We choose to made simulations on two compartments of white matter ( 2 fibers), because it gives most error than if we have different compartments as gray matter and cerebro-spinal fluid.

A simulation started by assigning the eigenvalues of the two Fibers. Then try to estimate them with the changment of the separation Angle between the two fibers. For each degree of Signal to noise ratio the following simulation procedures have been performed. The diffusion tensor in the principal coordinate was defined by assigning the eigenvalues to the diagonal elements of a $3 \times 3$ diagonal matrix (Ddiag). Its representation, D, in the laboratory frame was obtained by applying the rotation matrix associated with the orientation to the diagonal matrix [4].

The six $\mathrm{ADC}$ values along the directions defined by the diffusion tensor imaging scheme were determined from the

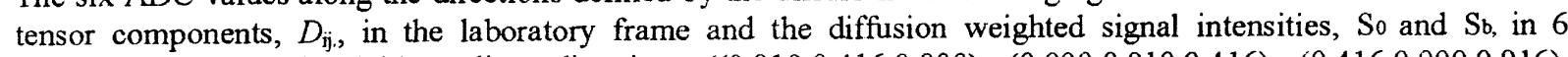
noncollinear magnetic field gradient directions $((0.910,0.416,0.000),(0.000,0.910,0.416),(0.416,0.000,0.916)$, $(0.916,-0.416,0.000),(0.000,0.916,-0.416),(-0.416,0.000,0.916))$ and their corres-ponding to $b$ values are $\left(250,500,750,1000,1250,1500,1750,2000 \mathrm{~s} / \mathrm{mm}^{2}\right)$ were computed from the known ADC and $b$ values with the changment of the separation angle between the 2 fibers [4]. White Gaussian distributed random noise (WGN $(0,1)$ ) was added to the ideal signal, but with modifications in the density to give the required value of the signal to noise ratio (SNR). The previous was made SNR used were 25,35 and $45 \mathrm{~dB}$ and no noise added signal . Noise perturbed signal intensities were used to recalculate the noise affected $A D C$ values [4]. The diffusion tensor components, $D_{\mathrm{ij}}$, and the Mean square attenuation Error were recalculated. At each SNR level 10000 replicate simulations were performed. The same was repeated with changing the gradient directions to 12,30 and 90 gradient directions ${ }^{23}$.

Experimental results were also obtained from data sets collected from a normal human volunteer on a 3T Siemens Trio system using a double spin-echo sequence with $8 \mathrm{~b}$-values spanning the range $[0,1500]$ at 30 directions. Both scans were repeated 4 times to investigate the effect of SNR. The total scan time for the 12-direction scan was approximately 30 minutes for the 30 -direction scan. The fractional anisotropy ${ }^{1}$ is computed for both components of the 2-tensor model to illustrate the convergence in 30-direction acquisitions. 


\section{RESULTS AND DISCUSSION}

Figures 1, 2, 3 and 4 shows the changment in the estimation error using the gradient algorithm. At no noise, as the number of gradients increase, the error in estimation increases. The error in Estimation is very high that decreases very slowly with the increase of the SNR. It is also clear that there no deviation in Estimates at low and high SNR.

Figures 5, 6, 7 and 8 shows the changment in the estimation error using the Differentiation algorithm. At no noise, as the number of gradients increase, the error in estimation increases. The error in Estimation and the standard deviation decreases with the increase of the SNR. It is also clear that instability in Estimation occurs at 6 and 12 directions, although they have better estimation than 30 and 90 gradient at no noise.

So, there must be some constraints in the starting point entering the conjugate gradient descent part to search minima to improve the algorithm.

Figures $9,10,11$ and 12 shows the changment in the estimation error using the Exhaustive algorithm. At no noise, as the number of gradients increase, the error in estimation increases. The error in Estimation and the standard deviation decreases with the increase of the SNR. For the Exhaustive Algorithm, In general, the 6 directions gives the least mean square error then the 12 gradient directions, we can direct the cause to the condition number of the gradient which were 1.00 for the 6 directions, 1.00 for 12 directions, 1.02 for 30 directions and 1.09 for 90 directions. Whish didn't appear in both Tuch et al. and differentiation algorithms.

As a general result for gradient algorithm, the error decreases with the increase of the separation angle, but for the differentiation and Exhaustive the error increases with the increasing of the separation angle. The error in Exhaustive algorithm is better than both differentiation and gradient algorithms.

For the differentiation method, the instability appears with the decrease of the SNR which isn't found in both the gradient descent and the Exhaustive because the computations in the differentiation are more than that of the Exhaustive method. We can say the same for the gradient algorithm. And generally, as increasing the number of gradient directions, the error in estimation decreases in the three algorithms.

\section{CONCLUSIONS}

We developed Two new methods for multi-tensor modeling the differentiation and the exhaustive algorithms, then we compared them with the gradient descent algorithm developed by Tuch [6], and finally, we used Monte Carlo simulations to estimate the effect of noise o their estimation.

For the gradient algorithm, the signal must be with more than $45 \mathrm{~dB}$ and the number of gradients be at least 12 directions to get least error and decrease the standard deviation in Tensors estimation. For the differentiation algorithm. The signal must be with more than $45 \mathrm{~dB}$ and the number of gradients be at least 30 directions to get least error and decrease the standard deviation in Tensors estimation And for the Exhaustive method ${ }^{15}$ to multiple exponential for the is straightforward, the computational complexity of the developed method can be shown to depend linearly on the number of components. The signal must be with at least $45 \mathrm{~dB}$ and the number of gradients is 12 directions to get least error and decrease the standard deviation in Tensors estimation. When extending the Gradient Algorithm to find more than two fibers, instability was found in the model simulations but the extension of the differentiation method the root-finding task of the third-degree polynomial is not as simple as with the seconddegree case. The extension for the exhaustive algorithm, the computation time is linear with the number of components. And no need for priori information for the calculations of tensors for both differentiation and Exhaustive algorithms. The differentiation method can be used to calculate the tensors faster, but the Exhaustive Algorithm is used to calculate the tensors more accurate fibers detection.

\section{REFERENCES}

[1] P. J. Basser, C. Pierpaout, "Microstructural and physiological features of tissues elucidated by quantitative diffusiontensor MRI." J. Magn. Reson.. vol. 111 (B). pp. 209-219. 1996

[2] M. E. Bastin, P. A. Armitage, I. Marshall, "A Theoretical Study of the effect of experimental noise on the measurement of anisotropy in Diffusion Imaging," Magn. Reson. Imag, vol. 16. pp. 773-785, 1998. 
[3] P.A. Armitage, M.E. Bastin, "Utilizing the diffusion-to-Noise ratio to optimize magnetic resonance diffusion tensor acquisition strategies for improving measurements of diffusion anisotropy," Magn. Reson. Med., vol. 45, pp.1056 $1065,2001$.

[4] Adam W. Anderson, "Theoretical Analysis of the Effects of Noise on Diffusion Tensor Imaging," Magn. Reson. Med.. vol 46, pp.1174-1188, 2001.

[5] P.J. Basser, J. Mattiello, D. LeBihan, "Estimation of the effective self-diffusion tensor from the NMR spin echo. J. Magn. Reson., vol. 103(B), pp. 247-254, 1994.

[6] D.S. Tuch, T.G. Reese, M.R. Wiegell, N. Makris, J.W. Belliveau, and V.J. Wedeen, "High angular resolution diffusion imaging reveals intravoxel while matter fiber heterogeneity," Magn. Reson. Med. vol.48, pp. 577-582, 2002.

[7] P.J. Basser and D.K. Jones, "Diffusion-tensor MRI: theory, experimental design and data analysis - a technical review," NMR Biomed. vol. 15, pp. 456-467, 2002.

[8] E.W. Hsu, D.L. Buckley, J.D. Bui, S.J. Blackband, and J.R. Forder, "Two-compartment diffusion tensor MRI of isolated perfused hearts," Magn. Reson. Med., vol.45, pp. 1039:1045, 2001.

[9] B.A. Inglis, E.L. Bossart, D.L. Buckley, E.D. Wirth III, and T.H. Mareci, "Visualization of neural tissue water compartments using biexponential diffusion tensor MRI," Magn. Reson.. Med., vol. 45, pp. 580-587, 2001.

[10] C.A. Clark, M. Hedehus, and M.E. Moseley, "Diffusion time dependence of the apparent diffusion tensor in healthy human brain and white matter disease," Magn. Reson. Med., vol. 45, pp. 1126-1129, 2001

[11] C.A. Clark, M. Hedehus, and M.E. Moseley, "In vivo mapping of the fast and slow diffusion tensors in human brain," Magn. Reson. Med., vol. 45, pp. 623-628, 2002.

[12] L.R. Frank, "Anisotropy in high angular resolution diffusion-weighted MRI," Magn. Reson. Med., vol. 45, pp. 935939, 2002.

[13] L.R. Frank, "Characterization of anisotropy in high angular resolution diffusion-weighted MRI," Magn. Reson. Med, vol. 47, pp. 1083-1099, 2002.

[14] W. Zhan, H. Gu, S. Xu, D. A. Silbersweig, E. Stern, Yi Yang, "Circular Spectrum Mapping for Intravoxel Fiber Structures Based on High Angular Resolution Apparent Diffusion Coefficients”, Magn. Reson. Med., vol. 49,pp. 1077$1088,2003$.

[15] Y. M. Kadah, X. Ma, S. LaConte, I. Yassine, X. Hu, "Robust multi-component modeling of diffusion tensor magnetic resonance imaging data", Proc. SPIE Medical Imaging 2005, Feb. 2005.

[16] J-D. Tournier, F. Calamante, A. Connelly, "Improved characterisation of crossing fibres: optimisation of spherical deconvolution parameters using a minimum entropy principle”, Proc. Intl. Soc. Mag. Reson. Med., vol. 13, pp. 384, 2005.

[17] J-D. Tournier, F. Calamante, A. Connelly, "Multiple fibre orientations estimated by spherical deconvolution: assessment of precision using the 'bootstrap' method", Proc. Intl. Soc. Mag. Reson. Med. , 2005:13 : 223.

[18] P.J. Basser, S. Pajevic, C. Pierpaoli, J. Duda, and A. Aldroubi, "In vivo fiber tractography using DT-MRI data," Magn. Reson. Med., vol. 44, pp. 625-632, 2000.

[19] S. Mori and P.C.M. van Zijl, "Fiber tracking: principles and strategies - a technical review," NMR Biomed. vol. 15, pp. $468-480,2002$.

[20] C. Poupon, C.A. Clark, V. Frouin, J. Regis, I. Bloch, D. Le Bihan, and J.-F. Mangin, "regularization of diffusion-based direction maps for the tracking of brain white matter fascicles," Neuroimage, vol. 12, pp. 184-195, 2000.

[21] R. Bammer, B. Acar, and M.E. Moseley, "In vivo tractography using diffusion imaging, "European J. Radiology , pp. $1-12,2002$.

[22] N.F. Lori, E. Akbudak, J.S. Shimony, T.S. Cull, A.Z. Snyder, R.K. Guillory and T.E. Conturo, "Diffusion tensor fiber tracking of human brain connectivity: acquisition methods, reliability analysis and biological results", NMR Biomed., vol. 15, pp. 493-515, 2002.

[23] S. Skare, M. Hedehus, M. E. Moseley, T. Qiang, "Condition Number as a Measure of Noise Performance of Diffusion Tensor Data Acquisition Schemes with MRI”, J. Magn. Reson. vol. 147, pp. 340-352, 2000. 


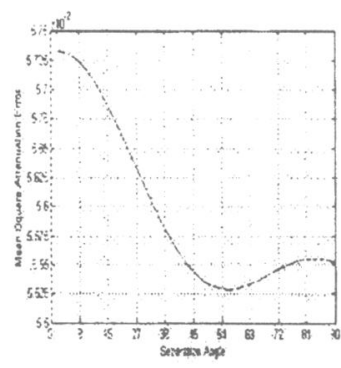

(a)

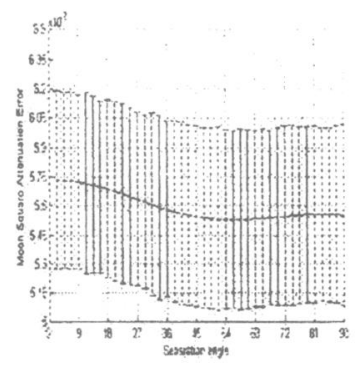

(b)

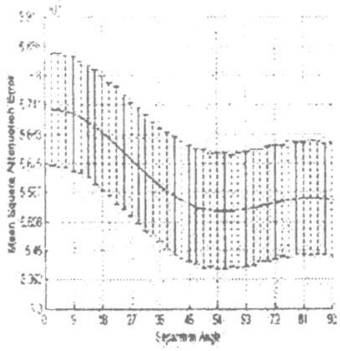

(c)

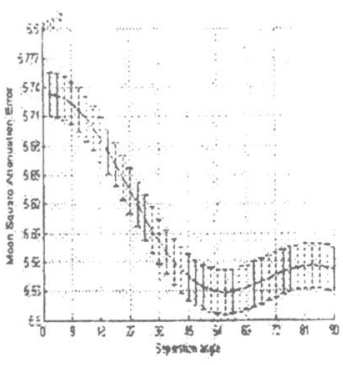

(d)

Figure 1: Estimated Attenuation Error in case of 6 gradient directions using the gradient algorithm at different SNR ((a) no noise, (b) $25 \mathrm{~dB}$, (c) $35 \mathrm{~dB}$, (d) $45 \mathrm{~dB}$ ).

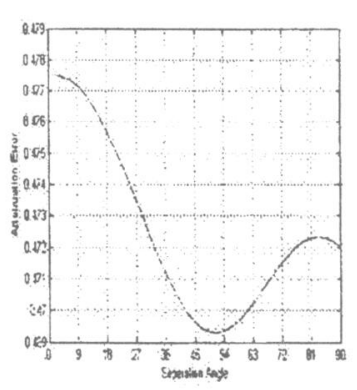

(a)

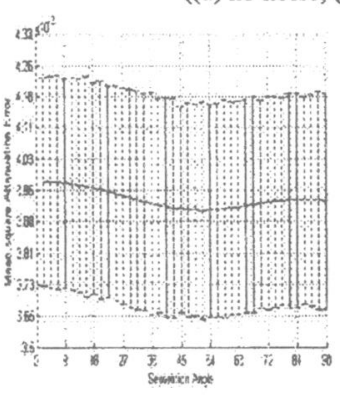

(b)

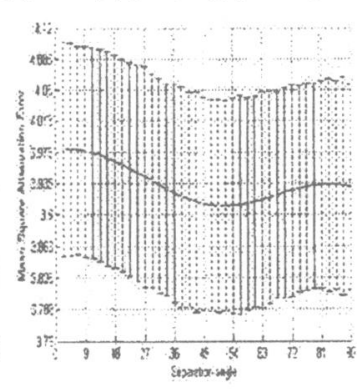

(c)

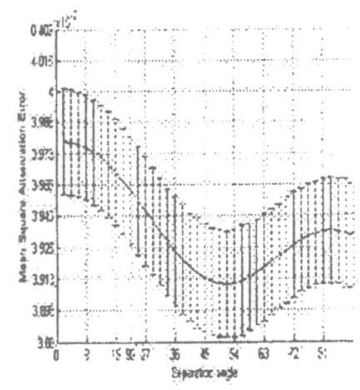

(d) ((a) no noise, (b) $25 \mathrm{~dB}$, (c) $35 \mathrm{~dB}$, (d) $45 \mathrm{~dB}$ ).
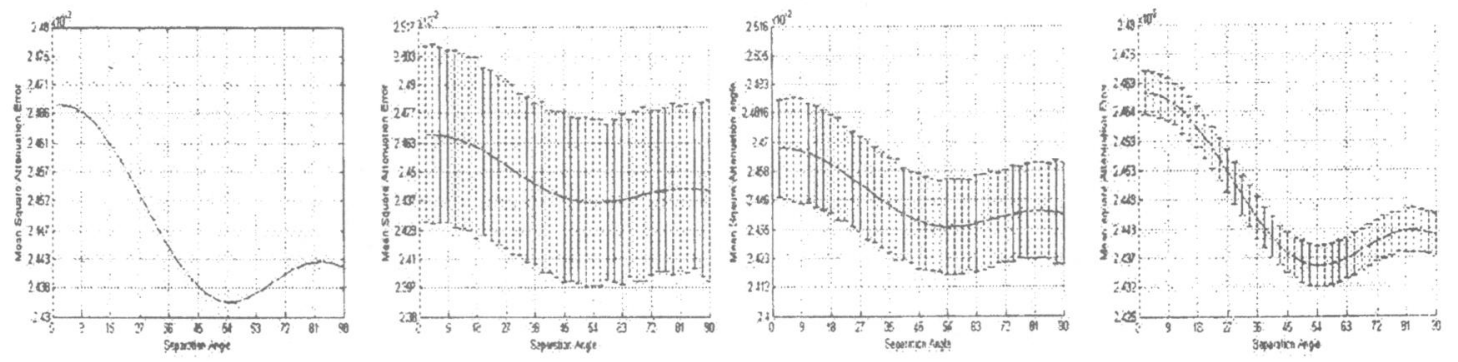

Figure 3: Estimated Attenuation Error in case of 30 gradient directions using the gradient algorithm at different SNR ((a) no noise, (b) $25 \mathrm{~dB},($ c) $35 \mathrm{~dB}$,(d) $45 \mathrm{~dB}$ ).

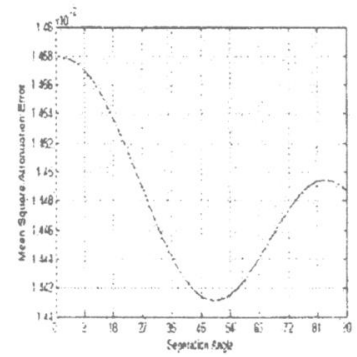

(a)

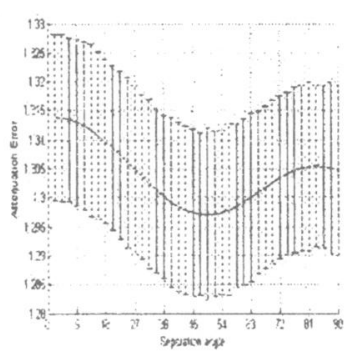

(b)

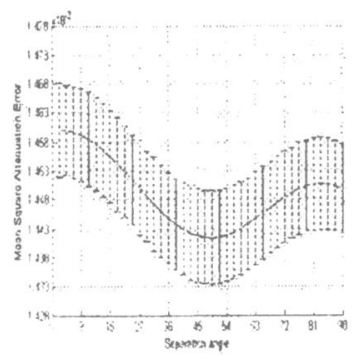

(c)

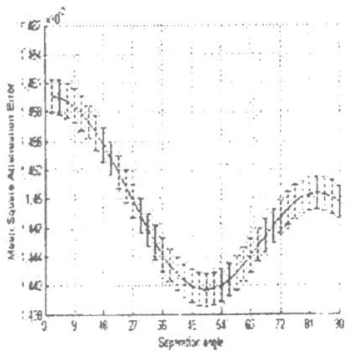

(d)

Figure 4: Estimated Attenuation Error in case of 90 gradient directions using the gradient algorithm at different SNR ((a) no noise, (b) $25 \mathrm{~dB}$, (c) $35 \mathrm{~dB}$.(d) $45 \mathrm{~dB}$ ). 


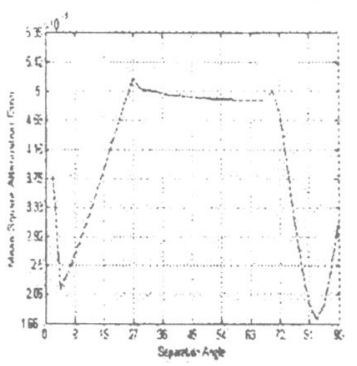

(a)

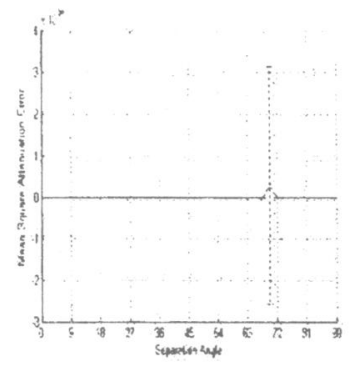

(b)

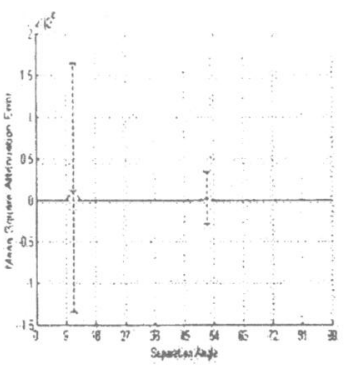

(c)

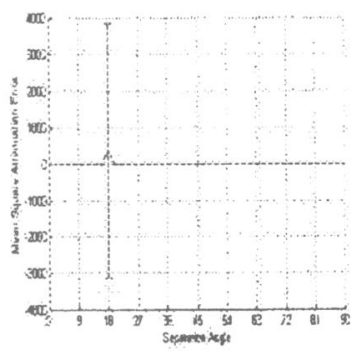

(d)

Figure 5: Estimated Attenuation Error in case of 6 gradient directions using the differentiation method at different SNR ((a) no noise, (b) $25 \mathrm{~dB}$, (c) $35 \mathrm{~dB}$, (d) $45 \mathrm{~dB}$ ).

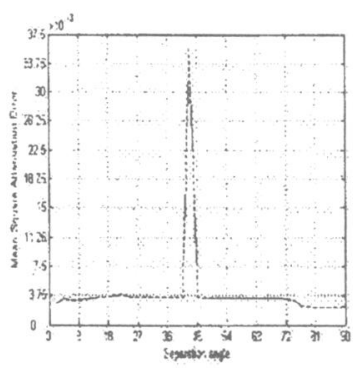

(a)

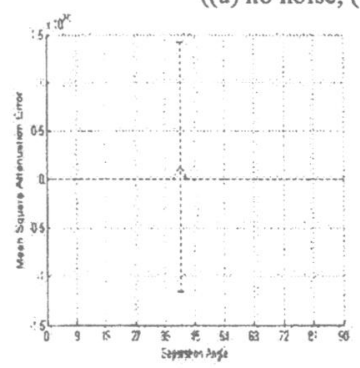

(b)

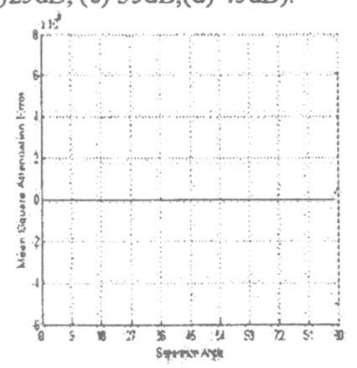

(c)

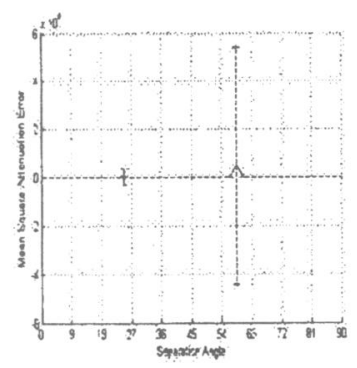

(d)

Figure 6: Estimated Attenuation Error in case of 12 gradient directions using the differentiation method at different SNR ((a) no noise, (b) $25 \mathrm{~dB}$, (c) $35 \mathrm{~dB}$, (d) $45 \mathrm{~dB}$ ).

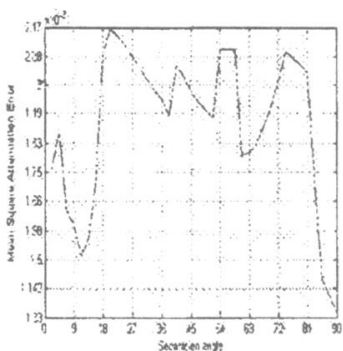

(a)

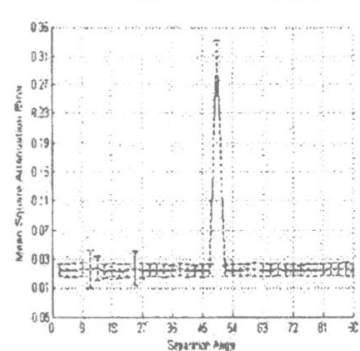

(b)

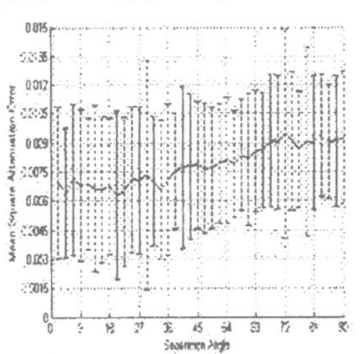

(c)

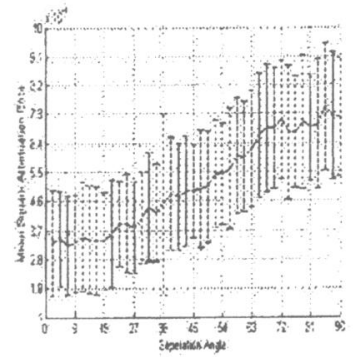

(d)

Figure 7: Estimated Attenuation Error in case of 30 gradient directions using the differentiation method at different SNR ((a) no noise, (b) $25 \mathrm{~dB}$, (c) $35 \mathrm{~dB}$,(d) $45 \mathrm{~dB}$ ).

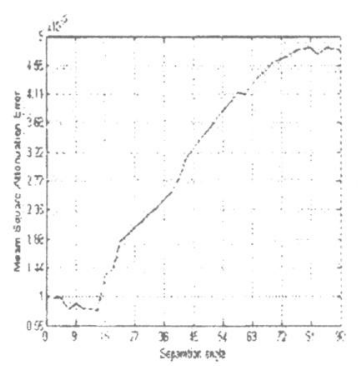

(a)

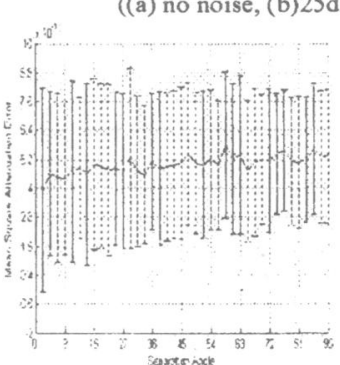

(b)

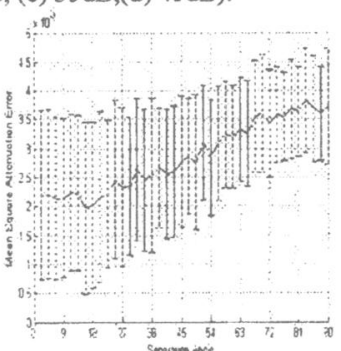

(c)

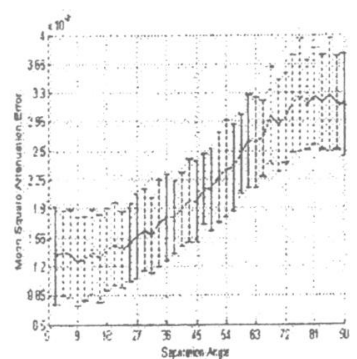

(d)

Figure 8: Estimated Attenuation Error in case of 90 gradient directions using the differentiation method at different SNR ((a) no noise, (b) $25 \mathrm{~dB}$, (c) $35 \mathrm{~dB}$,(d) $45 \mathrm{~dB}$ ). 


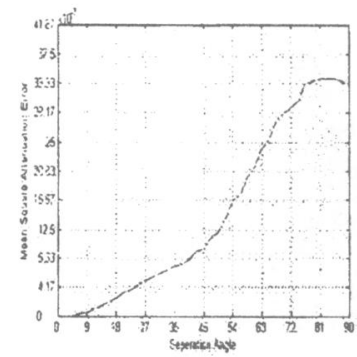

(a)

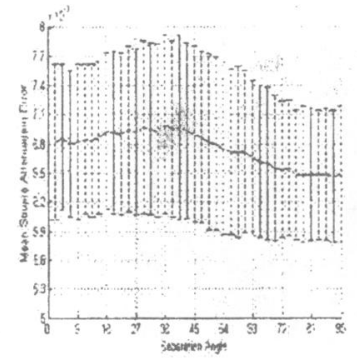

(c)

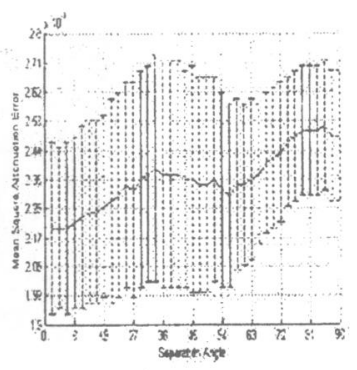

(d)

((a) no noise, (b) $25 \mathrm{~dB}$, (c) $35 \mathrm{~dB}$,(d) $45 \mathrm{~dB}$ ).

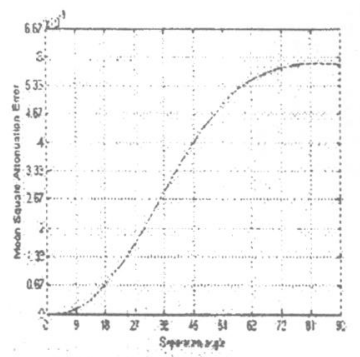

(a)

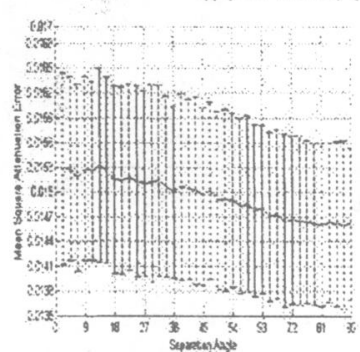

(b)

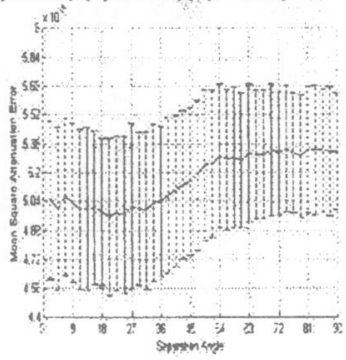

(c)

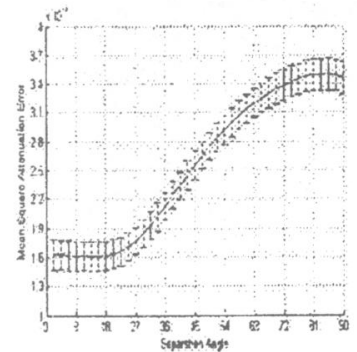

(d)

Figure 10: Estimated Attenuation Error in case of 12 gradient directions using the Exhaustive Algorithm at different SNR ((a) no noise, (b) $25 \mathrm{~dB}$, (c) $35 \mathrm{~dB}$, (d) $45 \mathrm{~dB}$ ).

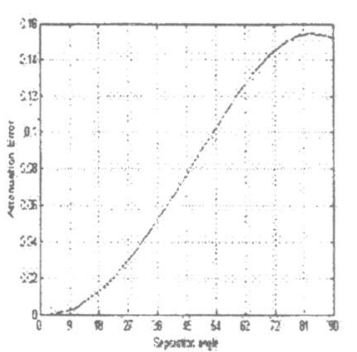

(a)

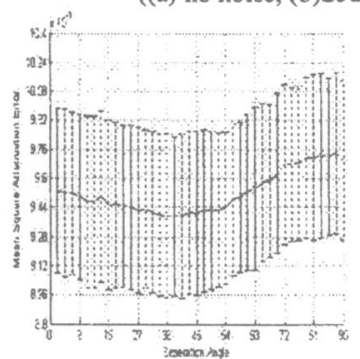

(b)

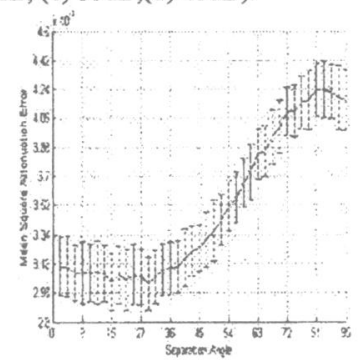

(c)

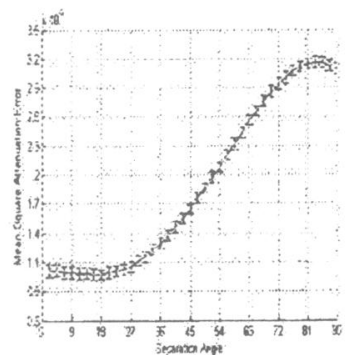

(d)

Figure 11: Estimated Attenuation Error in case of 30 gradient directions using the Exhaustive Algorithm at different SNR ((a) no noise, (b) $25 \mathrm{~dB}$, (c) $35 \mathrm{~dB}$, (d) $45 \mathrm{~dB}$ ).

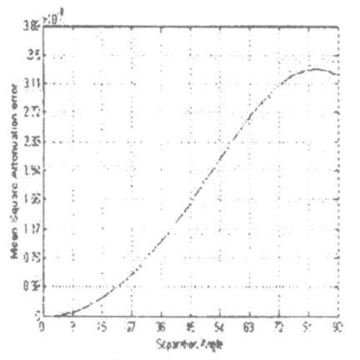

(a)

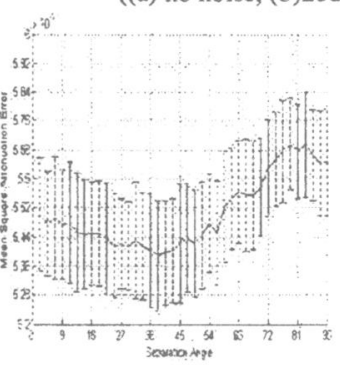

(b)

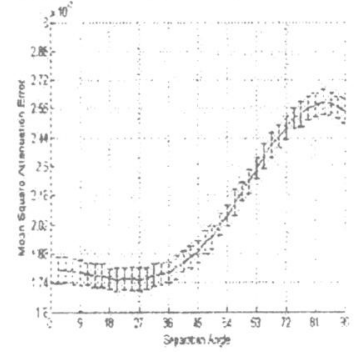

(c)

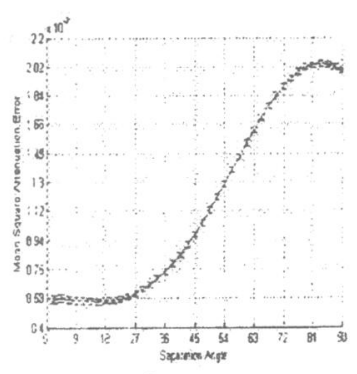

(d)

Figure 12: Estimated Attenuation Error in case of 90 gradient directions using the Exhaustive Algorithm at different SNR ((a) no noise, (b) $25 \mathrm{~dB}$, (c) $35 \mathrm{~dB}$.(d) $45 \mathrm{~dB}$ ). 


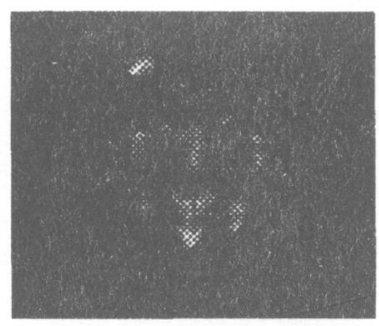

(a)

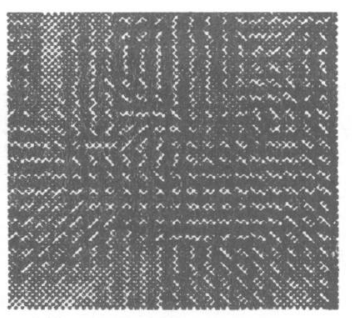

(b)

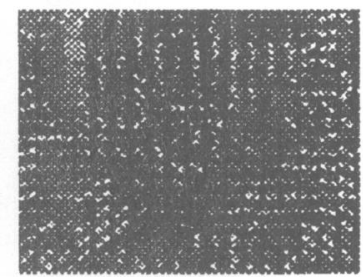

(d)

(e)

Figure 13: Estimated Tensors for the slice (a), Single Tensor Estimation (b), Two tensor Estimation using Gradient descent Algorithm (c), Differentiation Algorithm (d), Exhaustive Algorithm (e). 